\title{
Integer decomposition for polyhedra defined by nearly totally unimodular matrices
}

\author{
Dion Gijswijt*
}

May 23, 2005

\begin{abstract}
We call a matrix $A$ nearly totally unimodular if it can be obtained from a totally unimodular matrix $\tilde{A}$ by adding to each row of $\tilde{A}$ an integer multiple of some fixed row $a^{\top}$ of $\tilde{A}$. For an integer vector $b$ and a nearly totally unimodular matrix $A$, we denote by $P_{A, b}$ the integer hull of the set $\left\{x \in \mathbb{R}^{n} \mid A x \leq b\right\}$. We show that $P_{A, b}$ has the integer decomposition property and that we can find a decomposition of a given integer vector $x \in k P_{A, b}$ in polynomial time.

An interesting special case that plays a role in many cyclic scheduling problems, is when $A$ is a circular-ones matrix. In this case, we show that given a nonnegative integer $k$ and an integer vector $x$, testing if $x \in k P_{A, b}$ and finding a decomposition of $x$ into $k$ integer vectors in $P_{A, b}$ can be done time $O(n(n+m)+\operatorname{size}(x))$, where $A$ is an $m \times n$ matrix. We show that the method unifies some known results on colouring circular arc graphs and edge colouring nearly bipartite graphs. It also gives an efficient algorithm for a packet scheduling problem for smart antennas posed by E. Amaldi et al. in [1, 2].
\end{abstract}

Keywords: integer decomposition, totally unimodular, circular arc graph, nearly bipartite graph, cyclic scheduling, colouring.

\section{Introduction}

The main motivation for this paper is a very nice combinatorial problem from the field of telecommunications. This problem was posed by Amaldi, Capone and Malucelli in $[1,2]$ and concerns scheduling packets for smart antennas. In brief, the problem comes down to the following. Given a finite set $V$ of points on the unit circle and an angle $\alpha$, find a colouring of $V$ using a minimal number of colours. The colouring restriction is, that no segment of length $\alpha$ may contain more than 2 points of the same colour.

We show that this problem as well as some other colouring problems in the literature (see $[7,9,10,12])$ can be formulated as an integer decomposition problem for polyhedra defined by matrices that are very close to being totally unimodular. This motivates the following definitions. In the following, all vectors will be column-vectors. We call a matrix A nearly

*Department of Mathematics, University of Amsterdam, Plantage Muidergracht 24, 1018 TV Amsterdam, The Netherlands (gijswijt@science.uva.nl). 
totally unimodular if there exists a totally unimodular matrix $\left(\begin{array}{c}\tilde{A} \\ a^{\mathrm{T}}\end{array}\right)$ and an integer vector $c$, such that $A=\tilde{A}+c a^{\top}$. If $A$ is an $m \times n$ nearly totally unimodular matrix and $b \in \mathbb{Z}^{m}$ is a vector, we define the integer polyhedron $P_{A, b}$ by

$$
P_{A, b}:=\operatorname{conv} \cdot \operatorname{hull}\left(\left\{x \in \mathbb{Z}^{n} \mid A x \leq b\right\}\right) .
$$

In Section 2 we show that the polyhedron $P_{A, b}$ has the integer decomposition property. That is, every integer vector in $k P_{A, b}$ is the sum of $k$ integer vectors in $P_{A, b}$. The proof reduces the problem of decomposing an integer vector $x \in k P_{A, b}$ to a number of integer linear programs with totally unimodular constraint matrix $\left(\begin{array}{c}\tilde{A} \\ a^{\mathrm{T}}\end{array}\right)$. These can be solved in polynomial time by using the ellipsoid method (see [8]). However, in particular instances there may be a more efficient combinatorial algorithm to solve these linear programs. In Section 3 we consider the case where $A$ is a circular-ones matrix. We show that in this case a decomposition of $x$ can be found in time $O(n(n+m)+\operatorname{size}(x))$ when $A$ is an $m \times n$ matrix. In Section 4 we treat the packet scheduling problem in more detail and apply the results from Section 2 and 3 to obtain an efficient algorithm that solves the packet scheduling problem. We also give some applications to edge colouring nearly bipartite graphs and to colouring proper circular arc graphs.

\section{Integer decomposition}

Let $A$ be an $m \times n$ nearly totally unimodular matrix. We assume that $A$ is given as $A=\tilde{A}+c a^{\top}$ for an integer vector $c \in \mathbb{Z}^{m}$ and a totally unimodular matrix $\left(\begin{array}{c}\tilde{A} \\ a^{\top}\end{array}\right)$. Let $b \in \mathbb{Z}^{m}$ be an integer vector. A basic observation is the following.

Proposition 1. For any integer $s$, the polyhedron $P_{A, b} \cap\left\{x \in \mathbb{R}^{n} \mid a^{\top} x=s\right\}$ has the integer decomposition property.

Proof. First observe that since the matrix $\left(\begin{array}{c}\tilde{A} \\ a^{\mathrm{T}}\end{array}\right)$ is totally unimodular, the polyhedron

$$
P:=\left\{x \in \mathbb{R}^{n} \mid \tilde{A} x \leq b-s c, a^{\top} x=s\right\}
$$

is integer. Furthermore, by the well known theorem of Baum and Trotter characterizing totally unimodularity (see [3]), $P$ has the integer decomposition property. Since $P$ is integer, we have

$$
P \subseteq \text { int.hull }(P) \subseteq P_{A, b} \cap\left\{x \in \mathbb{R}^{n} \mid a^{\top} x=s\right\} \subseteq P,
$$

showing that $P=P_{A, b} \cap\left\{x \in \mathbb{R}^{n} \mid a^{\top} x=s\right\}$, which concludes the proof.

We can now prove that also $P_{A, b}$ has the integer decomposition property.

Theorem 1. Let $k$ be a nonnegative integer and let $x \in \mathbb{Z}^{n}$. Write $a^{\top} x=q k+r$ for integers $q$ and $r$ with $0 \leq r \leq k-1$. Then the following are equivalent:

(i) $x \in k P_{A, b}$, 
(ii) the system

$$
\begin{aligned}
A y & \leq r b \\
A(x-y) & \leq(k-r) b \\
a^{\top} y & =r(q+1)
\end{aligned}
$$

is feasible,

(iii) $x=x_{1}+x_{2}+\cdots+x_{k}$ for integer vectors $x_{1}, \ldots, x_{k} \in P_{A, b}$.

In particular, $P_{A, b}$ has the integer decomposition property.

Proof. It is clear that (iii) implies (i). To show that (i) implies (ii), suppose that $\frac{1}{k} x \in P_{A, b}$. Since the polyhedron $P_{A, b}$ is integer, we can write $\frac{1}{k} x=\frac{r}{k} x^{\prime}+\frac{k-r}{k} x^{\prime \prime}$, where $x^{\prime}, x^{\prime \prime} \in P_{A, b}$ and $a^{\top} x^{\prime}=q+1, a^{\top} x^{\prime \prime}=q$. Indeed, for a suitably large integer $M$ we can write

$$
M \cdot x=\sum_{i=1}^{k M} x_{i},
$$

where $x_{i} \in P_{A, b}$ and $a^{\top} x_{i} \in \mathbb{Z}$ for $i=1, \ldots, k M$ (since we can take the $x_{i}$ to be integer). Now take such a representation of $M \cdot x$ that minimizes

$$
\sum_{i=1}^{k M}\left(a^{\top} x_{i}\right)^{2}
$$

Then $\left|a^{\top} x_{i}-a^{\top} x_{j}\right| \leq 1$ for any $i$ and $j$, since otherwise we can replace $x_{i}$ and $x_{j}$ by $\lambda x_{i}+(1-$ $\lambda) x_{j}$ and $\lambda x_{j}+(1-\lambda) x_{i}$, where $\lambda=1 /\left|a^{\top} x_{i}-a^{\top} x_{j}\right|$ thus reducing (6). Hence $a^{\top} x_{i} \in\{q, q+1\}$ for each $i=1, \ldots, n$ and setting

$$
x^{\prime}:=\frac{1}{M} \sum_{i \mid a^{\top} x_{i}=q+1} x_{i} \quad \text { and } \quad x^{\prime \prime}:=\frac{1}{M} \sum_{i \mid a^{\top} x_{i}=q} x_{i}
$$

gives the required decomposition. It follows that $x^{\prime}$ satisfies (4).

To show that (ii) implies (iii), suppose that the system (4) is feasible. Observe that (4) is equivalent to

$$
\begin{aligned}
\tilde{A} y & \leq r(b-(q+1) c) \\
\tilde{A} y & \geq \tilde{A} x+(k-r)(q c-b) \\
a^{\top} y & =r(q+1) .
\end{aligned}
$$

Hence (4) has an integer solution $y$ because the matrix $\left(\begin{array}{c}\tilde{A} \\ a^{\mathrm{T}}\end{array}\right)$ is totally unimodular. Since $y$ is an integer vector in $r\left(P_{A, b} \cap\left\{x \in \mathbb{R}^{n} \mid a^{\top} x=q+1\right\}\right)$, we obtain by Proposition 1 a decomposition $y=y_{1}+\cdots+y_{r}$ of $y$ into $r$ integer vectors in $P_{A, b}$. Similarly, Proposition 1 gives a decomposition $x-y=x_{1}+\cdots+x_{k-r}$ of $x-y$ into $k-r$ integer vectors $x_{1}, \ldots, x_{k-r}$ in $P_{A, b}$. Hence $x=y_{1}+\cdots+y_{k}+x_{1}+\cdots+x_{k-r}$ is the required decomposition of $x$. 
From Theorem 1 it follows that testing membership of $P_{A, b}$ can be done in polynomial time, since checking feasibility of (4) can be done in polynomial time. Finding the required decompositions of $y$ and $x-y$ can be done in polynomial time. Indeed, denote

$$
P:=\left\{z \mid \tilde{A} z \leq b, a^{\top} z=s\right\} .
$$

Decomposing an integer vector $y \in r P$ can be done by solving $r-1$ linear programs, since a decomposition $y=y_{1}+y_{2}$ into integer vectors $y_{1} \in P$ and $y_{2} \in(r-1) P$ can be found by solving $\left\{\tilde{A} y_{1} \leq b, \tilde{A}\left(y-y_{1}\right) \leq(r-1) b, a^{\top} y_{1}=s\right\}$.

With a little more care (as was pointed out by an anonymous referee), a decomposition can be found in polynomial time as follows (see [6, 8]). First, $t \leq n+1$ affinely independent integer vectors $y_{1}, \ldots, y_{t} \in P$ and nonnegative numbers $\lambda_{1}, \ldots, \lambda_{t}$ with $\lambda_{1}+\cdots+\lambda_{t}=1$ can be found such that $\frac{1}{r} y=\lambda_{1} y_{1}+\cdots+\lambda_{t} y_{t}$ (algorithmic version of Carathéodory's theorem). Then $y^{\prime}:=y-\left\lfloor r \lambda_{1}\right\rfloor y_{1}-\cdots-\left\lfloor r \lambda_{t}\right\rfloor y_{t}$ is an integer vector in $r^{\prime} P$, where $r^{\prime}=r-\left\lfloor r \lambda_{1}\right\rfloor-\cdots-\left\lfloor r \lambda_{t}\right\rfloor<t$. Hence $y^{\prime}$ can be decomposed into $r^{\prime}$ integer vectors in $P$ by solving less than $t$ linear programs as above.

However, often $A$ and $b$ come from a combinatorial problem that allows more efficient ways of computing a decomposition of $x$. In the next section we discuss such a case, namely when $A$ is a circular-ones matrix.

\section{Circular-ones matrices}

Call a zero-one matrix $A$ a circular-ones matrix if in each row of $A$ the ones occur in circular consecutive order. That is, in each row the ones or the zeros form a contiguous block. Closely related is the circular-ones property (see [15]) for matrices. A matrix has the circular-ones property if it can be transformed into a circular-ones matrix by permuting the columns. If it exits, such a permutation can be found in linear time (see [5]). If $A$ is an $m \times n$ circular-ones matrix, then replacing each row $a^{\top}$ of $A$ in which the ones do not form a contiguous block, by $(\mathbf{1}-a)^{\top}$, we obtain an interval matrix, which is totally unimodular. Hence every circular-ones matrix is nearly totally unimodular. In this section we give an efficient algorithm for finding decompositions as in Theorem 1 in the special case of circular-ones matrices.

It will be convenient to use the following notation. For integers $i \leq j$, we denote the set $\{i, i+1, \ldots, j\}$ by $[i, j]$. For finite sets $U \subseteq V$ and $x \in \mathbb{R}^{V}$, we denote the characteristic vector of $U$ by $\chi^{U}$ and define $x(U):=x^{\top} \chi^{U}$.

If $P=\left\{x \mid A x \leq b, \mathbf{1}^{\top} x=s\right\}$, where $A$ is an interval matrix, and $b$ and $s$ are integer, decomposing an integer vector $x \in r P$ into $r$ integer vectors in $P$ can be done in polynomial time. In fact, such a decomposition can be found, that does not depend on the matrix $A$ or the vector $b$. In the case that $x$ is the characteristic vector of a subset $X \subseteq\{1, \ldots, n\}$, the decomposition simply amounts to colouring the $i$-th element of $X$ with colour $i$ modulo $r$. The proposed decomposition algorithm in Proposition 2 is not stongly polynomial, as it performs integer division on the coefficients of $x$. We will denote by size $(x)$ the encoding length of a given vector $x \in \mathbb{Z}^{n}$. 
Proposition 2. Let integers $s$ and $r>0$ and a vector $x \in \mathbb{Z}^{n}$ satisfying $\mathbf{1}^{\top} x=r$ s be given. Then we can find in time $O\left(n^{2}+\right.$ size $\left.(x)\right)$ a decomposition

$$
x=\sum_{t=1}^{l} n_{t} x_{t},
$$

of $x$ into integer vectors $x_{t}$ with $\mathbf{1}^{\top} x_{t}=s$ and such that for any interval $I \subseteq\{1, \ldots, n\}$ and any integer $d$ we have: $x(I) \leq r d \Rightarrow x_{t}(I) \leq d$ and $x(I) \geq r d \Rightarrow x_{t}(I) \geq d$, for each $t=1, \ldots, l$. The numbers $x_{t}$ are positive integers with $n_{1}+\cdots+n_{l}=r$ and $l \leq n+1$.

Proof. Define for $i=1,2, \ldots, n$ the integers $z_{i}, q_{i}$ and $r_{i}$ by:

$$
\begin{aligned}
& z_{i}:=x([1, i]) \\
& z_{i}=q_{i} r+r_{i} \quad \text { where } 0 \leq r_{i} \leq r-1 .
\end{aligned}
$$

Sort the elements of the set $\{0, r\} \cup\left\{r_{1}, r_{2}, \ldots, r_{n}\right\}$ in increasing order to obtain $0=r_{0}^{\prime}<$ $r_{1}^{\prime}<\ldots<r_{l}^{\prime}=r$. Now we define for $t=1,2, \ldots, l$ the numbers $n_{t} \in \mathbb{Z}_{+}$and vectors $x_{t} \in \mathbb{Z}^{n}$ by:

$$
\begin{aligned}
n_{t} & :=r_{t}^{\prime}-r_{t-1}^{\prime} \\
x_{t}([1, i]) & :=q_{i}+\delta_{r_{i} \geq r_{t}^{\prime}} \quad \text { for } \mathrm{i}=1, \ldots, \mathrm{n} .
\end{aligned}
$$

Here $\delta$ denotes the Kronecker delta attaining the value 1 if the subscript is true and the value 0 if it is false. It is an easy verification that the numbers $q_{i}$ and $r_{i}$ can be found in time $O\left(n+\operatorname{size}\left(x_{1}\right)+\cdots+\operatorname{size}\left(x_{n}\right)\right)$. Hence the $x_{t}$ and $n_{t}$ can be found in time $O\left(n^{2}+\operatorname{size}(x)\right)$.

Clearly $l \leq n+1, n_{1}+\ldots+n_{l}=r$ and $\mathbf{1}^{\top} x_{t}=s$ for each $t$. Since for $i=1, \ldots, n$

$$
\begin{aligned}
\sum_{t=1}^{l} n_{t} x_{t}([1, i]) & =\sum_{t=1}^{l}\left(r_{t}^{\prime}-r_{t-1}^{\prime}\right)\left(q_{i}+\delta_{r_{i} \geq r_{t}^{\prime}}\right) \\
& =r q_{i}+\sum_{t=1}^{l}\left(r_{t}^{\prime}-r_{t-1}^{\prime}\right) \delta_{r_{i} \geq r_{t}^{\prime}} \\
& =r q_{i}+r_{i} \\
& =x([1, i]),
\end{aligned}
$$

we have that $x=n_{1} x_{1}+\ldots+n_{l} x_{l}$. For any $t, t^{\prime}$ and any interval $[i, j]$ we have (defining $\left.q_{0}:=r_{0}:=0\right)$ :

$$
\begin{aligned}
\left|x_{t}([i, j])-x_{t^{\prime}}([i, j])\right| & = \\
\left|q_{j}-q_{i-1}+\delta_{r_{j} \geq r_{t}^{\prime}}-\delta_{r_{i-1} \geq r_{t}^{\prime}}-q_{j}+q_{i-1}-\delta_{r_{j} \geq r_{t^{\prime}}^{\prime}}+\delta_{r_{i-1} \geq r_{t^{\prime}}}\right| & = \\
\left|\delta_{r_{j} \geq r_{t}^{\prime}}-\delta_{r_{i-1} \geq r_{t}^{\prime}}-\delta_{r_{j} \geq r_{t^{\prime}}^{\prime}}+\delta_{r_{i-1} \geq r_{t^{\prime}}^{\prime}}\right| & \leq 1 .
\end{aligned}
$$

This implies the proposition. 
Theorem 2. Given an $m \times n$ matrix $A$ which is (up to multiplying some rows by -1) a circular-ones matrix, vectors $b \in \mathbb{Z}^{m}, x \in \mathbb{Z}^{n}$ and a nonnegative integer $k$, we can test in time $O(n(n+m))$ if $x \in k P_{A, b}$, and find in time $O(n(n+m)+$ size $(x))$ a decomposition

$$
x=\sum_{i=1}^{l} n_{i} x_{i},
$$

where $x_{i}$ is an integer vector in $P_{A, b}$ and $n_{i}$ is a positive integer for $i=1, \ldots, l$, the positive numbers $n_{i}$ satisfy $n_{1}+\cdots+n_{l}=k$ and $l \leq 2 n+2$.

Proof. Let $a=\mathbf{1}$ be the all-one vector of length $n$ and let $c \in\{-1,0,1\}^{m}$ be defined by $c_{i}=A_{i, n}$. Let $\tilde{A}:=A-c a^{\top}$. Then in each row of $\tilde{A}$ the nonzero elements form a contiguous block of either ones or minus ones. Write $a^{\top} x=q k+r$ as in Proposition 1. Now each of the inequalities in system (8) is of the form $y([i, j]) \leq d$ or $y([i, j]) \geq d$ for some integers $i, j \in\{1, \ldots, n\}$ and $d$. This implies that (8) can be solved by a shortest path algorithm as follows (see [10]). Construct a directed graph $D$ with vertex-set $\{0,1,2, \ldots, n\}$ and an arc $(i-1, j)$ of length $d$ for each inequality $y([i, j]) \leq d$, and an arc $(j, i-1)$ of length $-d$ for each inequality $y([i, j]) \geq d$. Now the system is feasible if and only if $D$ has no negative-length cycles. If there are no negative-length cycles, let $d_{i}$ be the length of a shortest-length path starting in vertex $i$ for $i=0,1,2, \ldots, n$. Then the vector $y \in \mathbb{Z}^{n}$ defined by $y_{i}:=d_{i}-d_{i-1}$ is an integer solution to (8). Detecting a negative-length cycle and finding the numbers $d_{i}$ can be done in time $O(n(n+m))$ by the Bellman-Ford method (see [4]). If an integer solution $y$ is found, we can by Proposition 2 find decompositions of $y$ and $x-y$ into integer vectors in $P_{A, b}$ in time $O(n(n+m)+\operatorname{size}(x))$.

\section{Applications}

In this section we discuss some applications of Theorems 1 and 2 .

\subsection{Proper circular-arc graphs}

For two points $a, b$ on the unit circle, the closed segment running clockwise from $a$ to $b$ is called an arc and is denoted by $[a, b]$. A proper circular arc system is a finite set of $\operatorname{arcs} A_{i}:=$ $\left[a_{i}, b_{i}\right], i=1, \ldots, n$ with the property that $A_{i} \not \subset A_{j}$ for any two distinct $i, j \in\{1, \ldots, n\}$. The proper circular arc graph $G$ associated with this system is the graph with vertex set $\{1, \ldots, n\}$, and two vertices $i$ and $j$ are joined by an edge if $A_{i} \cap A_{j} \neq \emptyset$. We will assume that the arcs are numbered in such a way that the points $a_{1}, a_{2}, \ldots a_{n}$ occur in clockwise order around the circle. For each $i=1, \ldots, n$, let $C_{i}:=\left\{j \in\{1, \ldots, n\} \mid a_{i} \in A_{j}\right\}$. Note that because no arc contains another arc, the ones in the characteristic vector of $C_{i}$ occur in circular consecutive order. Let $A$ be the $n \times n$ matrix with the characteristic vectors of the sets $C_{i}$ as rows. Then $A$ is a circular-ones matrix and $P_{\left(\begin{array}{c}A \\ -I\end{array}\right),\left(\begin{array}{l}\mathbf{1} \\ \mathbf{0}\end{array}\right)}$ is the stable set polytope of $G$. A $k$-colouring of $G$ corresponds to a decomposition of the all-one vector into $k$ integer vectors in the stable set polytope. As a corollary to Theorem 1, we find that the stable set polytope of a proper circular arc graph has the integer decomposition property. This result 
was proved by Niessen and Kind in [9]. By Theorem 2 we can find a colouring of $G$ using a minimum number of colours in time $O\left(n^{2} \log n\right)$ by binary search on the number of colours $k$. This is a result of Orlin, Bonucelli and Bovet (see [10]).

\subsection{A packet scheduling problem for smart antennas}

In recent years, there has been a growing interest in adaptive antenna arrays known as "smart antennas", for example for use in third generation mobile telecommunication systems (see $[11,14])$. A smart antenna may be viewed as a collection of co-located directive antennas in the plane that each transmit to (or receive from) a narrow beam (approximately 12 degrees). Each of these directive antennas can be independently oriented and can serve one user at a time. In order to avoid unwanted interference, there is a combinatorial restriction on the sets of users that can be served simultaneously: a user that is being served cannot be in the beam of a directive antenna that serves another user. This restricts the number of users that can be served during the same time slot. As an example, suppose that the angle of the beams from the directive antennas is 12 degrees and that three users are in a common sector of 12 degrees. If the middle of the three users is served, then the beam corresponding to the antenna that serves it must either contain the clockwise or the anticlockwise neighbour which therefore cannot be served at the same time. This implies that for a set of users that are served simultaneously, the angle between any of these users and it's second clockwise neighbour is more than 12 degrees. Hence the number of users that can be served in a single time slot is less than 60. In fact, we may assume that the number of available directive antennas is unlimited and that the sets of users that can be served simultaneously are determined exactly by this interference constraint.

In $[1,2]$ Amaldi and Malucelli considered the following two scheduling problems. Associate to each user a number representing its priority. The first scheduling problem is to find a set of users that can be served in a single time slot, maximizing the sum of their priority numbers. In [2] they gave a polynomial-time algorithm for this scheduling problem by reducing it to the problem of finding a maximum weight directed path in a weighted acyclic digraph. Here we will focus on the second scheduling problem.

Given a set of users, find a schedule for serving all the users, that needs a minimum number of time slots. That is, give a partition of the users into a minimal number of classes, where the users in each class can be served simultaneously.

Amaldi et al. devised heuristics for this problem and asked if the problem is NP-hard. As an application of Proposition 2 we will give an efficient afgorithm for solving this packet scheduling problem.

In the scheduling problem, the exact positions of the users are not needed, only their direction as seen from the smart antenna. Hence we can model the users by points on the unit circle and let the beams from the directive antennas correspond to arcs of a fixed length $\alpha$ of the unit circle. Following [2], the scheduling problem can be formalized as follows.

Let $\alpha \in(0,2 \pi)$ be given. A finite set $S$ of points on the unit circle will be called independent $t^{1}$ if there exist $|S| \operatorname{arcs}$ on the unit circle of length $\alpha>0$ such that each point in $S$ is in

\footnotetext{
${ }^{1}$ In [2] arcs are half-open segments, but for the definition, this is equivalent to using closed segments of the
} 
exactly one of these arcs and each of these arcs contains exactly one element of $S$. Note that any two of the $|S|$ arcs may intersect as long as the intersection does not contain a point in $S$. The independent sets correspond to the sets of users that can be served simultaneously. Given $\alpha$ and a finite subset $V$ of the unit circle (the users) the packet scheduling problem can now be restated as follows.

Partition problem. Given a finite subset $V$ of the unit circle and an $\alpha>0$, find a partition of $V$ into a minimal number of independent sets.

We will now show the connection between the partition problem and the circular-ones matrices. We make the following observation.

Observation. A finite set $S$ of points on the unit circle is independent if and only if $\left|S \cap\left[s, s^{\prime}\right]\right| \leq$ 2 for each arc $\left[s, s^{\prime}\right]$ of length $\alpha$ starting at a point $s \in S$.

Proof. To see necessity, suppose that some arc of length $\alpha$ contains $u, v, w \in S$ in this order, then any arc of length $\alpha$ containing $v$ also contains $u$ or $w$ and hence $S$ is not independent. For sufficiency, suppose that $\left|S \cap\left[s, s^{\prime}\right]\right| \leq 2$ for each arc $\left[s, s^{\prime}\right]$ of length $\alpha$ with $s \in S$. Let $v \in S$ and let $u$ and $w$ be the anticlockwise and clockwise neighbour in $S$ of $v$ respectively. The length of $[u, w]$ must be larger than $\alpha$ since $|[u, w] \cap S|>2$, and hence there exists an arc of length $\alpha$ intersecting $S$ only in $v$.

Note that the last argument also shows that given an independent set $S,|S|$ arcs of length $\alpha$ as in the definition of independent set, are easily constructed from $S$. Now given a finite subset $V$ of the unit circle, define for each $v \in V$ the row vector $a_{v} \in\{0,1\}^{V}$ as the incidence vector of the intersection of $V$ with the arc $\left[v, v^{\prime}\right]$ of length $\alpha$. Let $A x \leq b$ be the system consisting of the inequalities $a_{v} x \leq 2$ for $v \in V$, and the inequalities $\mathbf{0} \leq x \leq \mathbf{1}$. Then the matrix $A$ is (up to signs of the rows) a circular-ones matrix and the incidence vectors of the independent sets are precisely the integer vectors in $P_{A, b}$. Hence, the partition problem is to find a decomposition of the all-one vector into a minimal number of integer vectors in $P_{A, b}$. By Theorem 2 we can test if $V$ can be partitioned into $k$ independent sets in time $O\left(n^{2}\right)$. Hence using binary search on $k$, we obtain an $O\left(n^{2} \log n\right)$ algorithm for solving the packet scheduling problem.

\subsection{Edge colouring nearly bipartite graphs}

A graph $G$ is called nearly bipartite if we can obtain a bipartite graph by deleting a vertex from $G$. Let $G=(V, E)$ be a nearly bipartite graph and let $u \in V$ be a vertex of $G$ such that $G-u$ is bipartite with bipartition $V \backslash\{u\}=V_{1} \cup V_{2}$. Let $A$ be the $V \times E$ incidence matrix of $G$. Then $A$ is nearly totally unimodular. Indeed, let $a:=\chi^{F}$, where $F \subset E$ is the set of edges between $u$ and $V_{2}$, and define $\tilde{A}:=A-\chi^{\{u\}} a^{\top}$. Then $\left(\begin{array}{c}\tilde{A} \\ a^{\top}\end{array}\right)$ is the incidence matrix of a bipartite graph $G^{\prime}$ obtained from $G$ by splitting $u$ into two points. As the incidence matrix of a bipartite graph is totally unimodular, this implies that $A$ is nearly totally unimodular. It now follows by Theorem 1 that the matching polytope $P_{\left(\begin{array}{c}A \\ -I\end{array}\right),\left(\begin{array}{c}1 \\ \mathbf{0}\end{array}\right)}$ of

same length. 
$G$ has the integer decomposition property. Equivalently: the chromatic index of $G$ is the roundup of the fractional chromatic index. This result was proved in [7] and [12].

If $G$ is viewed as a multigraph by taking each edge $e$ with multiplicity $x_{e} \in \mathbb{N}$, then finding a $k$-edge colouring $G$ with $k$ minimal can be done as follows. First observe that we may assume that $k \geq \Delta$, where $\Delta$ is the maximum degree of a vertex in $G$ (counting multiplicities). Hence we have $q=0$ in Theorem 1 and $r=x(F)$ is the number of edges from $u$ to $V_{2}$. Solving system (4) amounts to finding an integer vector $y$ satisfying $\mathbf{0} \leq y \leq x$ and

$$
\begin{aligned}
y(e) & =0 \text { for } e \in \delta(u) \backslash F \\
y(e) & =x(e) \quad \text { for } e \in F \\
y(\delta(v)) & \leq x(F) \quad \text { for } v \in V \backslash\{u\} \\
y(\delta(v)) & \geq x(F)+x(\delta(v))-k \text { for } v \in V \backslash\{u\} .
\end{aligned}
$$

This can be done by reducing it to a flow problem with capacities and demands on the arcs. Hence we can find an integer solution $y$ (if it exists) in time $O(m n \log n)$ (see [13]). Since for $k \geq \Delta+x(F)$ we may take $y=\chi^{F}$, to find the minimal $k$, we need to check at most $O(\log x(F))$ values of $k$ using binary search. If an integer solution $y$ is found, decomposing $y$ and $x-y$ as in Theorem 1, comes down to capacitated edge colouring of the bipartite graphs $(V, E \backslash(\delta(u) \backslash F))$ and $(V, E \backslash F)$ respectively. This can be done in time $O\left(m^{2}\right)$ (see [13]).

When $x$ is a zero-one vector, the above algorithm comes down to the edge colouring algorithm as presented in [12].

\section{Acknowledgements}

The author would like to thank the anonymous referees for their many valuable suggestions, in particular for shortening the proof of the implication (i) $\Rightarrow$ (ii) in Theorem 1.

\section{References}

[1] E. Amaldi, A. Capone, F. Malucelli, Circular Arc Models and Algorithms for Packet Scheduling in Smart Antennas, IV ALIO/EURO Workshop on Applied Combinatorial Optimization, 2002.

[2] E. Amaldi, A. Capone, F. Malucelli, Discrete models and algorithms for packet scheduling in smart antennas, Proceedings second Cologne-Twente Workshop on Graphs and Combinatorial Optimization, Vol. 1, May 2003, 1-4.

[3] S. Baum, L.E. Trotter Jr., Integer rounding and polyhedral decomposition for totally unimodular systems, in: Otimization and Operations Research (Proc. Bonn 1977; R. Henn, B. Korte and W. Oettli, eds.), Lecture Notes in Economics and Math. Systems 157, Springer, Berlin, 1978, pp. 15-23.

[4] R.E. Bellman, On a routing problem, Quart. Appl. Math., no. 16 (1958), 87-90. 
[5] K.S. Booth, G.S. Lueker, Testing for the consecutive ones property, interval graphs, and graph planarity using $P Q$-tree algorithms, J. Comput. System Sci. 13 (1976), no. 3, 335379 .

[6] W. Cook, J. Fonlupt, A. Schrijver, An integer analogue of Carathéodory's theorem, J. Combin. Theory Ser. B 40 (1986), no. 1, 63-70.

[7] L. Eggan, M. Plantholt, The chromatic index of nearly bipartite multigraphs, J. Combin. Theory Ser. B 40 (1986), no. 1, 71-80.

[8] M. Grötschel, L. Lovász, A. Schrijver, Geometric Algorithms and Combinatorial Optimization, Springer-Verlag, Berlin, 1988.

[9] T. Niessen, J. Kind, The round-up property of the fractional chromatic number for proper circular arc graphs, J. Graph Theory 33 (2000), no. 4, 256-267.

[10] J.B. Orlin, M.A. Bonuccelli, D.P. Bovet, An $O\left(n^{2}\right)$ algorithm for colouring proper circular arc graphs, SIAM J. Algebraic Discrete Methods 2 (1981), no. 2, 88-93.

[11] A. Perez-Neira, X. Mestre, J.R. Fonollosa, Smart antennas in software radio base stations, IEEE Communications Magazine, Vol. 39 (2), Feb. 2001, 166-173.

[12] B. Reed, Edge coloring nearly bipartite graphs, Oper. Res. Lett. 24 (1999), 11-14.

[13] A. Schrijver, Combinatorial optimization. Polyhedra and efficiency. Vol A., SpringerVerlag, Berlin, 2003.

[14] K. Sheikh, D. Gesbert, D. Gore, A. Paulraj, Smart antennas for broadband wireless access networks, IEEE Communications Magazine, Vol. 37 (11), Nov. 1999, 100-105.

[15] A. Tucker, Matrix characterizations of circular-arc graphs, Pacific J. Math., 39 (1971), $535-545$. 